\title{
A Rare Case Report of Siblings with Infantile and Late Infantile Forms of Neuronal Ceroid Lipofuscinoses
}

\author{
Ghosh $\mathbf{M}^{1}$, Chowdhury $\mathrm{J}^{2}$, Das $\mathrm{S}^{3}$, Banerjee $\mathbf{M}^{4}$, \\ Chakraborty $\mathrm{S}^{5}$
}

${ }^{1}$ Dr. Moumita Ghosh, MBBS, MD Paediatric Medicine, RMO-cum Clinical Tutor, Medical College Kolkata, West Bengal,India, ${ }^{2}$ Dr. Jasodhara Chowdhury, MBBS, MD Paediatric Medicine, Senior Resident, Medical College Kolkata, West Bengal India, ${ }^{3} \mathrm{Dr}$. Shubhadeep Das, MBBS, MD Paediatric Medicine, RMO-cum Clinical Tutor, Chittaranjan Sishu Sadan, Kolkata, West Bengal, India, ${ }^{4}$ Dr. Mukut Banerjee, MBBS, MD Paediatric Medicine, RMO-cum Clinical Tutor, Burdwan Medical College, West Bengal, India, ${ }^{5}$ Dr. Swapna Chakrabory, MBBS, MD Paediatric Medicine, Professor, Medical College Kolkata West Bengal, India.

\section{Introduction}

$\mathrm{N}^{\mathrm{e}}$ euronal Ceroid Lipofuscinoses (NCL) is a neurodegenerative disorder predominantly involving gray matter. There is lysosomal accumulation of auto fluorescent ceroid and lipofuscin in cells with degeneration of cerebral and cerebellar cortex and retina. Inheritance is autosomal recessive. Incidence is $1.6 / 100,000$ live births ${ }^{1}$. The 4 classic forms are Infantile type, Late Infantile type, Juvenile type and Adult type. We present a case of 3 siblings of unrelated parents where the eldest had Infantile form of NCL, the second child had Late Infantile Form, and the third child is normal. Both children having different phenotypic presentations have curvilinear inclusion bodies on EM of axillary skin biopsy, low levels of tripeptidyl amino peptidase 1(TPP1) and normal levels of palmitoyl protein thioesterase 1(PPT1), a finding classically present in the Late Infantile variety involving CLN2 mutation. After extensive search we failed to find a case report describing a similar finding.

\section{The Case}

A 4 years old female child was admitted in our hospital with status epilepticus. She was born out of non-consanguineous marriage and delivered at term with an uneventful pre and perinatal period. She was

\author{
Address for correspondence \\ Dr. Moumita Ghosh \\ E-mail:dr.moumitaghosh@gmail.com
}

\begin{abstract}
Neuronal Ceroid Lipofuscinoses is a neurodegenerative disorder predominantly involving grey matter. The 4 classic forms are Infantile type, Late Infantile type, Juvenile type and Adult type. We present a case of 3 siblings of unrelated parents where the eldest had Infantile form of NCL, the second child had Late Infantile Form, and the third child is normal. Both children having different phenotypic presentations have curvilinear inclusion bodies on EM of axillary skin biopsy, low levels of tripeptidyl amino peptidase 1(TPP1) and normal levels of palmitoyl protein thioesterase 1(PPT1), a finding classically present in the Late Infantile variety involving CLN2 mutation. After extensive search we failed to find a case report describing a similar finding.
\end{abstract}

apparently normal till 3 years of age when she developed recurrent seizures, loss of speech and cognitive delay. She also developed unsteady gait followed by frequent falls, and clumsiness of hand movements. Gradually she was unable to stand and sit. On examination, she was completely bedridden. There was generalized hypotonia, deep reflexes were normal and plantar reflex was bilateral flexor. Myoclonic jerks and generalised tonic clonic seizures (GTCS) was present. There was microcephaly (head circumference $42 \mathrm{~cm}$ ) but facial dysmorphism and organomegaly was absent. Skin and hair was normal. Ophthalmoscopy revealed bilateral optic atrophy and absence of cherry red spot. MRI brain showed cerebral and cerebellar atrophy. EEG showed a slow background with a giant occipital spike on photic stimulation at 2-4 Hz. The Electro Retinogram (ERG) was abnormal and Visual Evoked Potential (VEP) showed prolonged latency and diminished amplitude. Electron microscopy (EM) of axillary skin biopsy showed curvilinear inclusion bodies and enzyme analysis revealed low level of tri peptidyl amino peptidase $1(3.1 \mathrm{nmol} / \mathrm{hr} / \mathrm{mg}$ protein) and normal level of palmitoyl protein thioesterase $(72 \mathrm{nmol} / \mathrm{hr} / \mathrm{mg}$ protein). Thus she was diagnosed as a case of Late Infantile NCL.

How to cite this article?

Ghosh M, Chowdhury J, Das S, Banerjee M, Chakraborty S. A Rare Case Report of Siblings with Infantile and Late Infantile Forms of Neuronal Ceroid Lipofuscinoses. J Nepal Paediatr Soc 2013;33(3):220-222. 


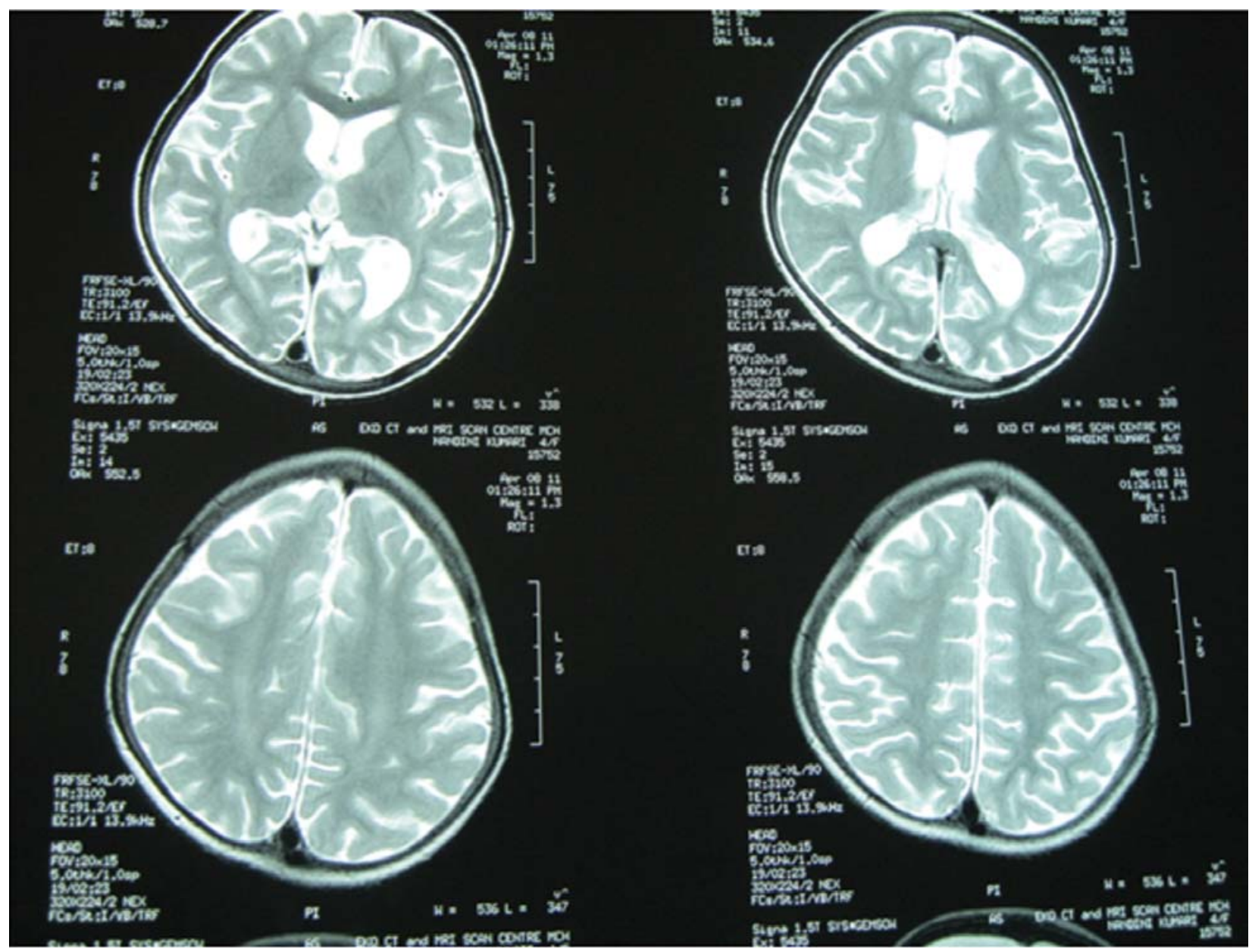

Fig 1: Showing cerebral atrophy in Magnetic Resonance Imaging of the brain

The child had an elder brother who was also delivered at term. Pre and perinatal course was uneventful. The developmental milestones were normal till 8 months age when he developed recurrent seizures. Over next 2 years he could no longer sit, use his hands, and recognize his parents. Feeding the child became exceedingly difficult. He expired at the age of 3 years. He had microcephaly and bilateral optic atrophy. There was absence of facial dysmorphism and organomegaly. The MRI, ERG and VEP reports suggested NCL. Interestingly, Electron microscopy (EM) of axillary skin biopsy showed curvilinear inclusion bodies and enzyme analysis revealed low level of tri peptidyl amino peptidase $1(2.4 \mathrm{nmol} / \mathrm{hr} / \mathrm{mg}$ protein, normal $85-326 \mathrm{nmol} /$ $\mathrm{hr} / \mathrm{mg}$ protein) and normal level of palmitoyl protein thioesterase (55nmol/hr/mg protein, normal $20-93 \mathrm{nmol} /$ $\mathrm{hr} / \mathrm{mg}$ protein). Thus, the child was a case of Infantile $\mathrm{NCL}$ but with EM and enzyme levels suggestive of CLN2 mutation seen in Late Infantile NCL.

There was a 2 year old younger sibling who was asymptomatic. The physical examination, ophthalmological evaluation and enzyme analysis of the third sibling was normal.

\section{Discussion}

$\mathrm{NCL}$ is characterized by seizures, predominantly myoclonic, also GTCS and akinetic seizures, loss of cognition and skills, ataxia, visual impairment and premature death within 6-10 years. Optic atrophy, macular degeneration and abnormal ERG occur in all 3 types preceding visual symptoms. VEP shows giant waves initially (except Juvenile type), later amplitude is diminished. Neuroimaging shows progressive cerebral and cerebellar atrophy. Cerebellar involvement is particularly severe in Late Infantile type. EEG shows slow background with high amplitude polyphasic spikes in the occipital region on photic stimulation at 2-4 $\mathrm{Hz}$. EM of brain, rectum, axillary skin and conjunctiva show characteristic inclusion bodies ${ }^{2}$. Recently, enzyme assays and molecular genetics have diminished the need for invasive procedures and made prenatal diagnosis a possibility.

The Infantile form has onset within 1 year of age and follows an acute course. Granular osmiophilic inclusion bodies are found in EM. There is mutation in genes encoding a lysosomal enzyme, palmitoyl protein 
thioesterase 1, with locus CLN1 on chromosome 1p32. Mutation in CLN1 have also resulted in Late Infantile, Juvenile ${ }^{3}$, and Adult phenotypes ${ }^{4}$. Cases of congenital $\mathrm{NCL}$ have been reported due to c.764dupA mutant cathepsin $D^{5}$.

Late Infantile is the second most common type, originates between 2-4 years, and follows an acute course. Seizures, loss of cognition and ataxia occur early, visual loss occurs late. The classical findings on EM are curvilinear bodies. It occurs due to mutations in CLN2 gene encoding a lysosomal enzyme tripeptidyl peptidase 1 with locus on chromosome 11p15. Mutations have also been noted in the CLN5, CLN6, and CLN8 genes that code for integral membrane proteins that have not been completely characterized.

The Juvenile form is most common, with onset between 5-10 years age, and follows a chronic course. Visual and behavioral problems predominate. Seizures occur later in the course. The ultrastructural findings are fingerprint profiles. Genetic mutations occur in CLN3 on chromosome 16p12.1.

In our case, the parents had a non-consanguineous marriage and both Infantile Form and Late Infantile forms of NCL were present in children of the same parents. Both affected children have curvilinear inclusion bodies on EM of axillary skin biopsy, low levels of tripeptidyl amino peptidase 1 and normal palmitoyl protein thioesterase 1 , a finding usually present in the Late Infantile variety involving CLN2 mutation. After extensive search we failed to find a case report describing a similar finding. Patients presenting with Juvenile NCL but having low levels of palmitoyl protein thioesterase or palmitoyl protein thioesterase are said to have variant Infantile and Late Infantile NCL respectively but there are no reports of dissimilar presentations in the same family. We found a case report of dissimilar neuropsychiatric presentations of two siblings with Juvenile $\mathrm{NCL}^{6}$ but genetic and enzymatic studies were not done in their case.

\section{Conclusion}

In conclusion, the highlight of this case report is two siblings of unrelated parents having different clinical presentations of NCL, namely Infantile and Late Infantile forms, with EM and enzyme analysis report suggestive of Late Infantile NCL. After extensive search we have failed to find any other case report showing a similar finding.

\section{References}

1. Al-Bloushi MAS, Anim JT, Habeeb YK. Neuronal Ceroid Lipofuscinoses: Report of five cases in Kuwait. Kuwait Med J 2006;38 (4):315-20.

2. Farrell DF, Sumi SM. Skin punch biopsy in the diagnosis of juvenile neuronal ceroid-lipofuscinosis. Arch Neurol 1977;34:39-44.

3. Wisniewski KE, Kida E, Connel F, Zhong N. Neuronal ceroid lipofuscinoses research update. Neurol Sci 2000;21:S49-56.

4. Van Digglen OP, Thobois S, Tilikete C, et al. Adult Neuronal Ceroid Lipofuscinosis with PalmitoylProtein Thioesterase Deficiency: First adult-onset patients of a childhood disesase. Ann Neurol 2001; 50:269-272.

5. Siintola E, Partanen S, Strömme P, Haapanen A, Haltia M, Maehlen J, Lehesjoki AE, Tyynelä J. Cathepsin $D$ deficiency underlies congenital human neuronal ceroid-lipofuscinosis. Brain 2006;129(Pt 6):1438-45.

6. Lee TS, Poon SH, Chang P. Dissimilar neuropsychiatric presentations of two siblings with juvenile neuronal ceroid lipofuscinosis (Batten disease). J Neuropsychiatr Clin Neurosci 2010 Winter;22(1):123.E14-5. 